\title{
LARGE-DEFLECTION STACKED MULTI-ELECTRODE ELECTROSTATIC ACTUATOR
}

\author{
Hanseup S. Kim, Asli B. Ucok, and Khalil Najafi \\ Center for Wireless Integrated MicroSystems (WIMS ERC) \\ University of Michigan, Ann Arbor, Michigan 48109-2122
}

\begin{abstract}
This paper presents a large-deflection electrostatic actuator that is comprised of multiple stacked flexible electrodes. This actuator can provide: 1) a large deflection at low voltages due to a small gap between electrodes; and 2) a modal operation of a single electrostatic device with ' $n$ '-different center deflections in an ' $n$ '-layered electrostatic device. The actuator obtains the full deflection by allowing the top flexible electrode to bend consecutively to the next electrodes until reaching the bottom. The top electrode can also be forced to stop at different deflection heights by proper application of voltages selectively to the first few electrodes.

The resultant micro-fabricated actuator achieved a large out-of-plane deflection $(6.09 \mu \mathrm{m})$ of a top Parylene layer at a low voltage (less than $50 \mathrm{~V}$ ), which is a considerable reduction, more than $30 \%$, compared to a conventional single electrode actuator. Using three flexible Parylene electrodes, the fabricated actuator showed three different modes of deflections: $1.00 \mu \mathrm{m}, 2.66 \mu \mathrm{m}$, and $6.09 \mu \mathrm{m}$. The fabricated actuator operated for more than $1,000,000$ cycles continuously in a 'touch' mode, where each electrode physically bent and contacted the next electrodes. Although the actuator successfully operated such a long period, it showed signs of stress relaxation and fatigue of polymer electrodes. In atmosphere, the actuator response time is $<91 \mathrm{~ms}$, which was the minimum detectable limit of the equipment.
\end{abstract}

Key Words: Large Deflection, Stacked Multi-layer actuator, Electrostatic actuator

\section{INTRODUCTION}

Diaphragms play a major role in MEMS actuators because they are efficient in volume displacement, offer large area bending, and are easy to fabricate using lithography techniques. Diaphragm based actuators are used to generate fluid flows through micro channels [1] and applied in micro-mirrors for optical applications. [2, 3]. They can be deflected in a controlled manner using various transduction techniques, such as electrostatic [4, 5], electromagnetic [6], piezoelectric [7-9], and thermo-pneumatic [10] actuation.

Electrostatic actuation has many advantages over others in deflecting a diaphragm. It generally uses low power due to its capacitive operation. Even in micro domain, it can generate a large amount of force. Moreover, it is more compatible with CMOS-based processes than electromagnetic and piezoelectric actuation due to its simple structure and its generous material selectivity. In addition, it responds faster than thermopneumatic actuation, and is more immune to temperature change than piezoelectric actuation. Therefore, various applications, such as micro pumps and micro mirrors, have adopted electrostatic actuation for their membrane deflection.

Conventional varying-gap electrostatic actuators have a fairly small maximum out-of-plane (vertical) deflection limited by the gap spacing between the two electrodes. The gap cannot be easily increased since it significantly increases the operating voltage. Thus, obtaining a large maximum deflection without increasing the required voltage is a challenge in electrostatic actuators.

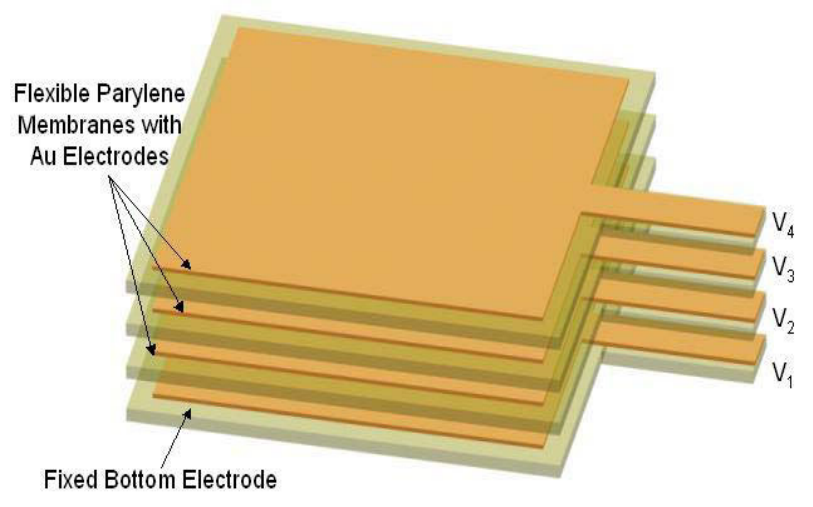

Figure 1. 3D-illustration of stacked multi-layer electrostatic actuator: The bottom electrode is fixed to the substrate while remaining electrodes are on flexible Parylene membranes. Modal deflection depends on both the number of flexible membranes used and the voltages applied to the corresponding electrodes.

To overcome this challenge, curved electrodes can be used [11] to reduce the effective distance between the two capacitive electrodes around the perimeter of the actuator. However, this requires a more complicated fabrication process. The stacked multi-electrode actuator structure reported in this paper reduces the operating voltage and increases the total deflection by stacking up multiple, freestanding, flexible polymer membrane electrodes on top of each other with small air gaps in between, as illustrated in Figure 1. The small gaps between membrane/electrode pairs enable the top membrane to deflect down to the next layer at a low voltage, and then this pair is pulled down to the layer below. This process repeats to the following layers underneath in a similar way. Thus, the stacked multiple electrode actuators achieve a large deflection of the top membrane with a relatively lower voltage than conventional electrostatic actuators. In addition, the multiple electrode actuators can have a modal operation of multiple deflections by selectively bending the top membrane to certain layers only.

\section{STACKED MULTI-ELECTRODE ACTUATOR}

\section{Large deflection at a low voltage}

The operation of the multi-electrode electrostatic actuator is similar to a conventional capacitive type electrostatic actuator in that alternative signals are applied to adjacent layers to create attractive forces between them. This force will pull the layers towards each other. In conventional devices one of the two electrodes is fixed. In the stacked actuator all electrodes move towards each other except for the bottom electrode that is fixed. 
To find the pull-in voltage (the voltage at which a diaphragm collapses) of the multi-electrode actuator, we start from the mechanical restoring force and the electrical force equations:

$$
\begin{aligned}
& F_{\text {Electrical }}=\frac{\varepsilon_{o}}{2} \cdot \text { Area } \cdot \frac{V^{2}}{(d-w)^{2}} \\
& F_{\text {Restoring }}=k \cdot w
\end{aligned}
$$

where $\varepsilon_{o}$ is the dielectric constant for air, $V$ is an applied electrical voltage, $d$ is an initial distance between two parallel plates, $w$ is a deflection distance, and $k$ is a spring constant.

In the multi-electrode actuator, since both electrodes move equally instead of only one, $w$ is replaced by $2 w$ in equation 1 , and $k$ is replaced by $2 k$ in equation 2 . This results in a pull-in voltage value that remains the same as an actuator with a fixed electrode, as shown in equation 3:

$$
V=\sqrt{\frac{8 \cdot k \cdot d^{3}}{27 \cdot \varepsilon_{o} \cdot \text { Area }}}
$$

Therefore, the multi-electrode actuator will have the same pull-in voltage between any electrodes including the fixed bottom electrode. A multi-electrode actuator can obviously have a smaller pull-in voltage by reducing the gap distance, $d$.

\section{Modal operation}

To obtain different deflection distances of the top membrane for multi-deflection modes from a single device, signals can be applied to some of layers selectively, as illustrated in Figure 2. Generally, when 'n' layers are used, 'n' different deflections are achievable in a modal operation of the multi-electrode actuator.

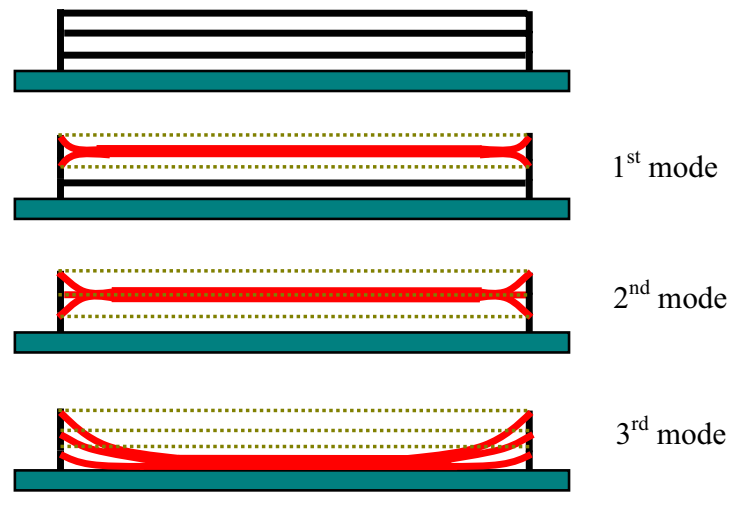

Figure 2. Illustration of three modal deflections from a threelayered electrostatic actuator

\section{FABRICATION}

A stacked multi-electrode actuator has been designed and fabricated. The actuator has three freestanding flexible Parylene membranes, each coated with thin metal films. Each actuator is a $100 \mu \mathrm{m} \times 100 \mu \mathrm{m}$ square, with a total height of $\sim 15.7 \mu \mathrm{m}$, and an average gap of $\sim 2.2 \mu \mathrm{m}$ between individual electrodes. A total of three suspended membranes are used, with the bottom electrode being fixed and on the substrate.

At the beginning, an array of different sizes of singleelectrode actuators is formed in order to identify their structural strength to generate a multi-electrode actuator structure, as shown in Figure 3.

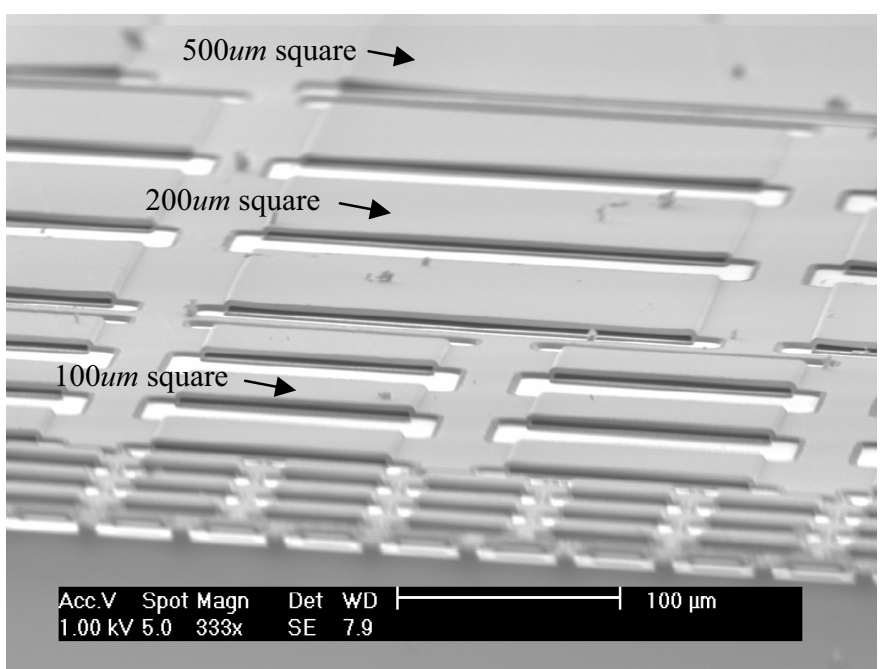

Figure 3. Microfabricated array of single electrode electrostatic actuator: up to a $200 \mu \mathrm{m}$ square size, single freestanding Parylene membranes were successfully formed.

Then, the multi-electrode structure is formed by stacking up multiple sets of sacrificial photoresist of AZ1827 $(2.7 \mu \mathrm{m})$, thin Parylene layer $(2 \mu \mathrm{m})$, and conductive thin gold layer $(0.3 \mu \mathrm{m})$, as shown in Figure 4. After patterning of each sacrificial layer photoresist, it is hard baked at $110^{\circ} \mathrm{C}$ for 30 minutes to create a sloped profile around the edges of the photoresist and provide good step coverage for subsequent metal layers. The hard baked photoresist layers are removed in acetone leaving air-gaps between layers after two sides of Parylene sidewalls have been dry-etched by oxygen flow. The completed actuator is naturally dried to form a stacked multi-electrode electrostatic actuator without stiction, as shown in Figure 5.

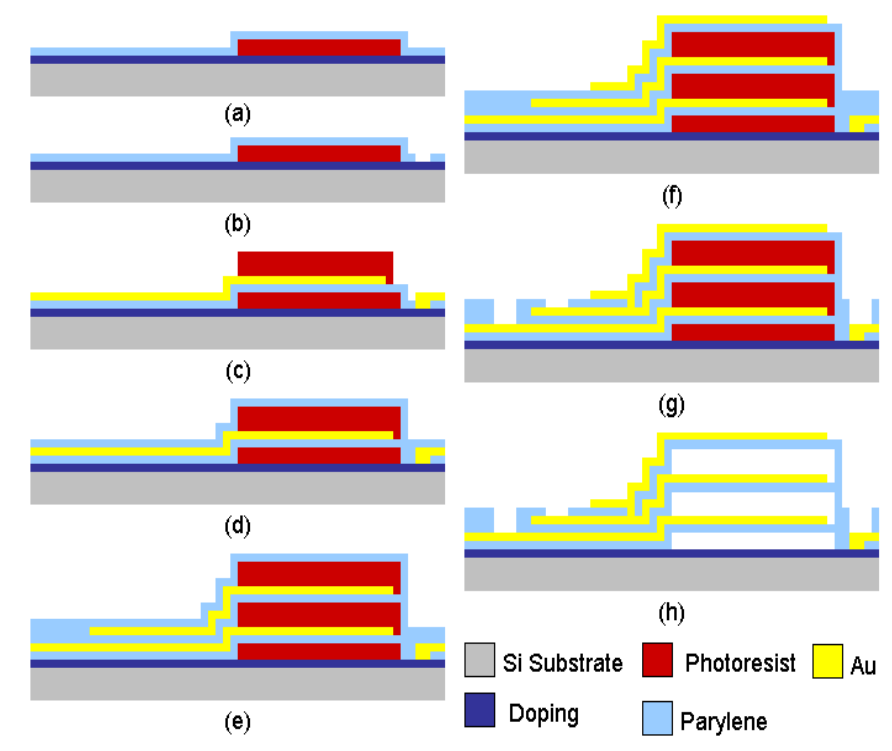

Figure 4. The process flow for the fabrication of large deflection stacked multi-layer electrostatic actuator utilizing simple photoresist sacrificial layer and acetone dissolution.

The completed devices are mounted and interconnected to electronic packages. Originally each device is wire-bonded through each device pad that is located on each layer of multiple Parylene stacks. However, each Parylene layer provides too soft surface for a wire-bonding of the electrical pads. Thus, at the electrical pads, a 
conductive epoxy, P10, is added to complete the connectivity of wire-bonding from the actuators to outside packages, as shown in Figure 6.

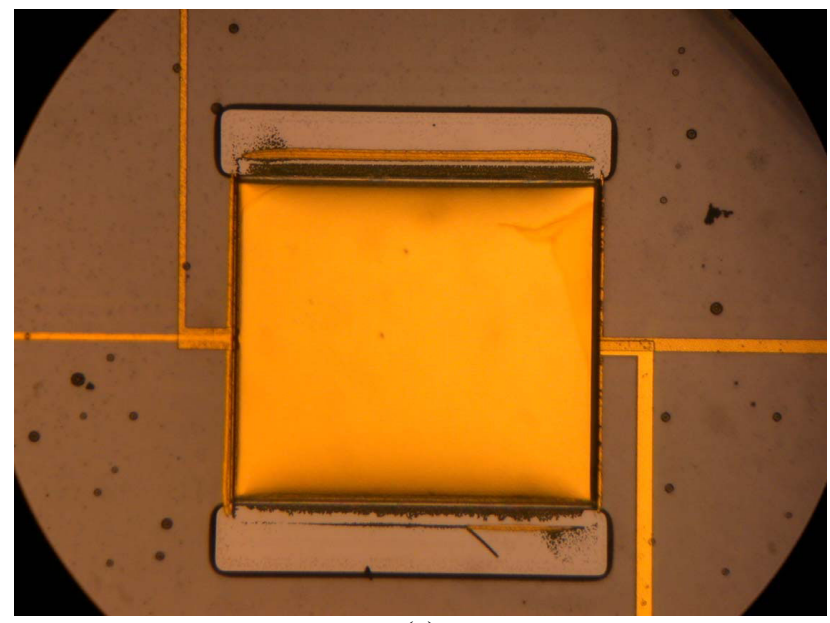

(a)

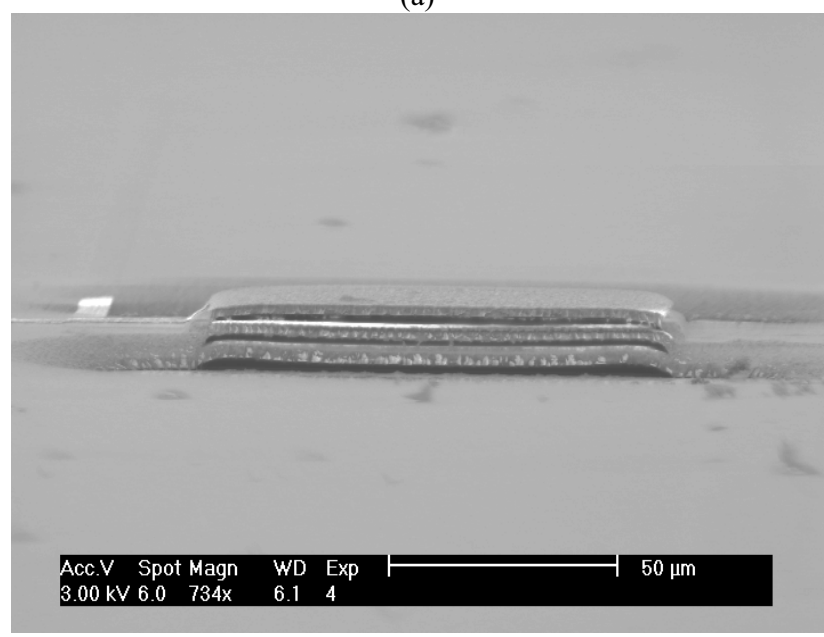

(b)

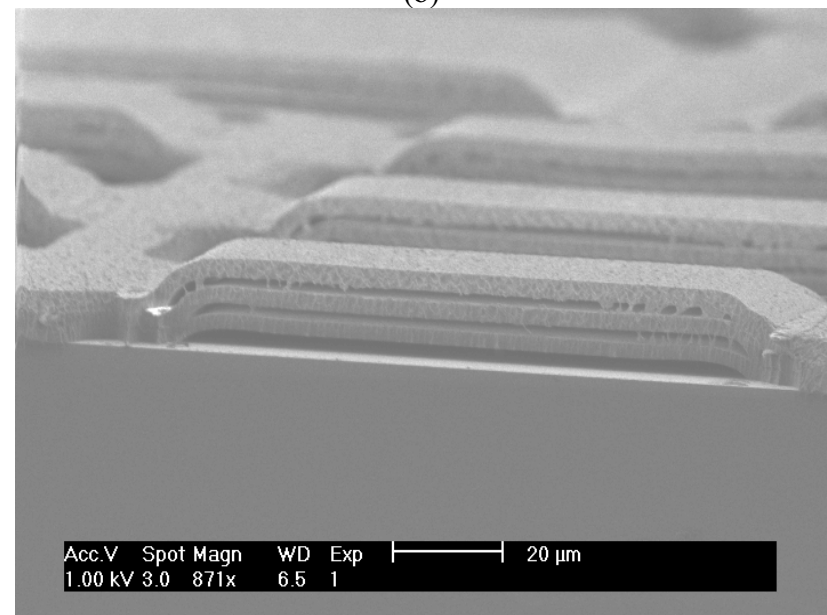

Figure 5. Microfabricated stacked multi-electrode (three) electrostatic actuator: a) Top view of the completed actuator, b) Side View (SEM), and c) Side view (SEM) of an array of multi-electrode actuator.

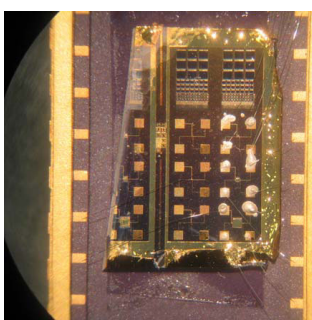

(a)
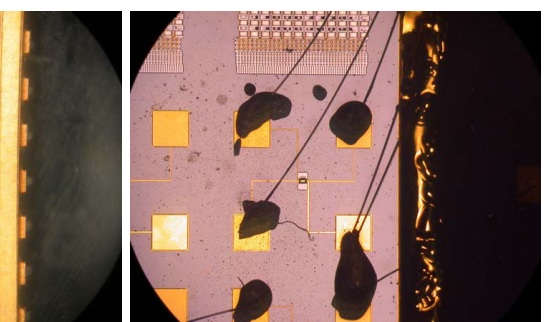

(b)
Figure 6. Wire-connected multi-electrode electrostatic actuators: a) a piece of actuator die on electronic package wire-bonded, and b) a close-up view of a multi-electrode actuator and interconnections. Conductive epoxy is additionally used for complete interconnection to outside package.

\section{TEST RESULTS}

The fabricated actuators were packaged and then tested using both AC and DC drive signals, as discussed below. First, a full DC deflection was observed through Zygo interferometer by looking at the fringe changes on the actuator surface. Then, the deflection was measured by observing the capacitance change using a HP4284A LCR meter. The maximum deflection happened at DC $13 \mathrm{~V}$ in the beginning stage, however, it was observed that this pull-in voltage drifted up to more than $130 \mathrm{~V}$ as DC signal is continually applied. The maximum deflection caused a $10.776 \mathrm{pF}$ capacitance change in total. This high capacitance change was due to 1) the fact that the bottom electrode was directly connected the silicon wafer itself, which caused a great deal of parasitic values; and 2) the fact that the metal residue outside membrane areas hanged out and touched membranes' metal layers.

Second, the response time of the actuator was measured by applying 0 volts to 30 volts through the LabView 7.0 software. Here, the 30 volts was applied to make sure the diaphragm fully collapsed in a specific case. The response time was $\sim 91 \mathrm{~ms}$, as shown in Figure 6 . The response time was symmetric for both membrane collapse and release. The response time was measured in atmosphere where air was squeezed out from the space between electrodes through $\sim 2.2 \mu \mathrm{m}$ gaps. Faster operation is possible in a vacuum for use in reflective optical display applications.

Third, a lifetime reliability test of the actuator was performed. The actuator was tested by supplying DC signal between 0 volts and 30 volts at $10 \mathrm{~Hz}$. The test was performed more than 24 hours, and resulted in a significant decrease of capacitance change per cycle over time, as shown in Figure 7. The overall decrease of capacitance change was more than $50 \%$ after one million cycles. This decrease is possibly due to 1) the stretching of a visco-elastic polymer material, Parylene; 2) the stress charge (stress relaxation) of the Parylene membrane due to heats generated by repeated motions; or 3 ) the charging effect on the Parylene membrane caused by continual DC voltages. Over time, the polymer membrane stretches and provides a smaller restoring force resulting in smaller moving distance and, thus, smaller capacitance change over time.

Finally, the modal operation was tested by applying voltages selectively to some layers. The modal operation was successfully measured through a Zygo interferometer, as seen in Figure 8. The modal operation resulted in distinctive deflection differences: $1^{\text {st }}$ mode of $1.00 \mu \mathrm{m}, 2^{\text {nd }}$ mode of $2.66 \mu \mathrm{m}$, and $3^{\text {rd }}$ mode (full collapse) of $6.09 \mu \mathrm{m}$. 


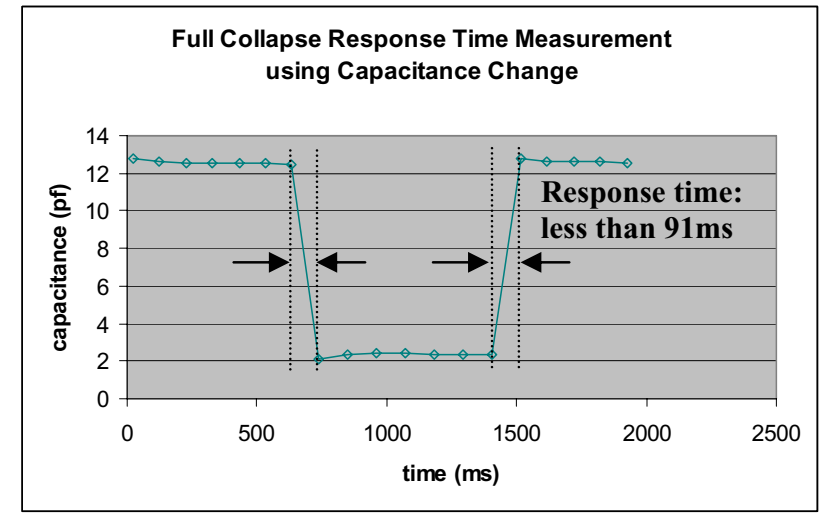

Figure 6. Response time measurements of a multi-electrode electrostatic actuator

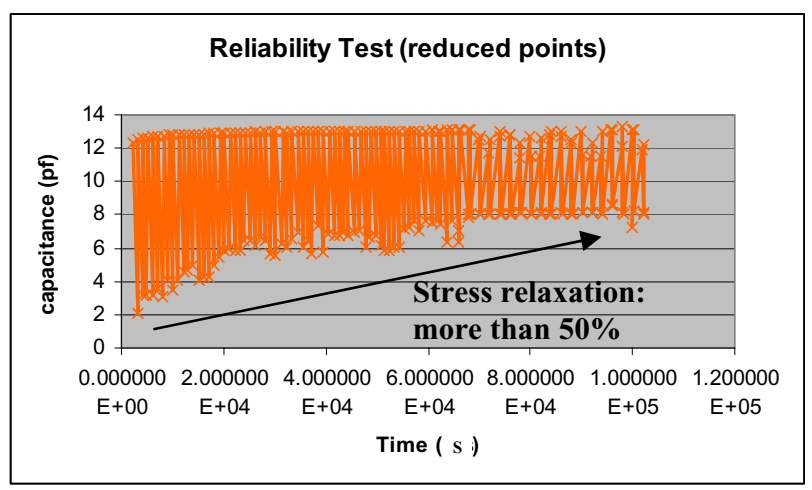

Figure 7. Capacitance change measurement for a reliability test of a multi-electrode electrostatic actuator

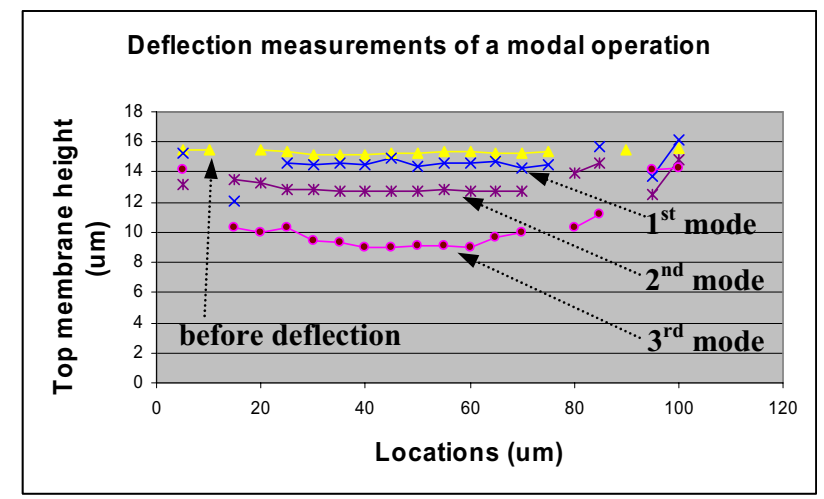

Figure 8. Modal deflection measurement of a multi-electrode electrostatic actuator

\section{DISCUSSIONS AND CONCLUSIONS}

The stacked multi-electrode electrostatic actuator has demonstrated that it can achieve a large out-of-plane deflection at a low voltage and can be operated in several different deflection modes. The actuator can be used in a variety of applications, including optical devices. For this purpose, the Parylene multi-electrode actuator has shown great potential. First, Parylene can be conformally deposited with precise thickness. Moreover, this flexible Parylene film is used in a simple surface micro-machining fabrication process with a good yield. Second, the actuator can have very high fill factor in an array form when used as a display pixel. This is more feasible especially because the actuator's membrane has an intrinsic tensile stress. Third, the actuator is fabricated under $110^{\circ} \mathrm{C}$.
Thus, the actuator can be added on top of CMOS circuitry through a post-CMOS process.

Several observations and issues were also made during testing. First, frequent failures were observed during multi-layer full collapsing. The failure happened when some of the multi-electrodes did stick together without collapsing to the next layers further. In this case, the failure resulted in much higher operating voltages to move those membranes into the fully-collapsed positions. This implies that further design improvements like corrugation may be needed to facilitate collapse regardless of membrane position. Second, Parylene showed signs of fatigue and stress relaxation over time, and this led to a drift of the pull-in voltage and deflection distance as well as response time. Third, the surface roughness of Parylene membrane was measured by a surface profiler, Datek, showing up to $3000 \AA$ of roughness. This is higher than expected and needs to be improved. Fourth, DC charging was observed. Combined with Parylene membrane's stress relaxation, the charging effect caused the pull-in voltage to increase to a very high value (more than $150 \mathrm{~V}$ ). An attempt to eliminate the charging effect in an $80^{\circ} \mathrm{C}$ oven failed because it caused membranes to touch and stick together due to tiny gaps between. Further work is necessary in this area.

\section{ACKNOWLEDGEMENT}

The authors thank Joseph Potkay for valuable help on measurements. This project is funded by the Engineering Research Centers Program of the National Science Foundation under Award Number EEC-9986866. Travel support has been generously provided by the Transducers Research Foundation and by the DARPA MEMS and DARPA BioFlips programs.

\section{REFERENCES}

[1] R. Zengerle, S. Kluge, M. Richter, and A. Richter, "A bidirectional silicon micropump," MEMS '95, Netherlands, pp. 19-24, 1995.

[2] D. M. Bloom, "Grating light valves for high resolution displays," Electron Devices Meeting, Technical Digest., International, pp. 343, 1994.

[3] L. J. Hornbeck, "Current status of the digital micromirror device (DMD) for projection television applications," Electron Devices Meeting. Technical Digest., International, pp. 381-384, 1993.

[4] Cabuz-C, Herb-WR, Cabuz-EI, and Son-Thai-Lu, "The dual diaphragm pump," MEMS 2001., Interlaken, Switzerland, pp. 519-22, 2001.

[5] R. J. Linderman, O. Nilsen, and V. M. Bright, "The resonant micro fan gas pump for active breathing microchannels," pp. 1923-1926, 2003.

[6] C. H. Ahn and M. G. Allen, "Fluid Micropumps Based on rotary magnetic actuators," MEMS '95, pp. 408-412, 1995.

[7] T. Gerlach, "Pumping gases by a silicon micro pump with dynamic passive valves," 1997 International Conference on Solid-State Sensors and Actuators, Chicago, pp. 357-360, 1997.

[8] K.-P. Kamper, J. Dopper, W. Ehrfeld, and S. oberbeck, "A selffilling low-cost membrane micropump," MEMS '98, pp. 432437, 1998.

[9] A. Olsson, G. Stemme, and E. Stemme, "The first valve-less diffuser gas pump," MEMS '97, Nagoya, Japan, pp. 108-113, 1997.

[10] C. Grosjean and Y.-C. Tai, "A thermopneumatic peristaltic micropump," The 10th International Conference on Solid State Sensors, Actuators, and Microsystems, Sendai, Japan, pp. 1999.

[11] H. S. Kim, K. Najafi, P. D. Washabaugh, and L. P. Bernal, "Large-deflection out-of-plane electrostatic buckled-electrode actuator," Transducers '03, Boston, pp. 794-797, 2003. 